\title{
TAB3 upregulates Survivin expression to promote colorectal cancer invasion and metastasis by binding to the TAK1-TRAF6 complex
}

\author{
Chen Luo ${ }^{1,2, *}$, Rongfa Yuan ${ }^{1,2, *}$, Leifeng Chen ${ }^{1,2}$, Wei Zhou ${ }^{3}$, Wei Shen ${ }^{1}$, Yumin Qiu ${ }^{1,2}$, \\ Jun Shao ${ }^{1,2}$, Jinlong Yan $^{1,2}$ and Jianghua Shao ${ }^{1,2}$ \\ ${ }^{1}$ Department of General Surgery, Second Affiliated Hospital of Nanchang University, Nanchang 330006, China \\ ${ }^{2}$ Jiangxi Province Key Laboratory of Molecular Medicine, Nanchang 330006, China \\ ${ }^{3}$ Department of Gastrointestinal Surgery, Jiangxi Provincial Cancer Hospital, Nanchang 330029, China \\ *These authors contributed equally to this work \\ Correspondence to: Jianghua Shao, email: shao5022@163.com \\ Keywords: TAB3; Survivin; NF-KB pathway; colorectal cancer; metastasis \\ Received: August 02, $2017 \quad$ Accepted: October 28, $2017 \quad$ Published: November 18, 2017 \\ Copyright: Luo et al. This is an open-access article distributed under the terms of the Creative Commons Attribution License 3.0 \\ (CC BY 3.0), which permits unrestricted use, distribution, and reproduction in any medium, provided the original author and source \\ are credited.
}

\section{ABSTRACT}

Transforming growth factor- $\beta$-activated kinase 1 (TAK1)-binding protein 3 (TAB3) is involved in cancer proliferation and metastasis, but its role in colorectal cancer remains unclear. In this study, we demonstrated that TAB3 is upregulated in colorectal cancer tissues and that high TAB3 levels correlated with tumor metastasis and a poor prognosis in colorectal cancer. In addition, TAB3 knockdown decreased Survivin expression and suppressed colorectal cancer cell migration and invasion in vitro, and reduced liver metastasis in vivo. Importantly, we found that TAB3 regulated Survivin expression by activating the NF-KB pathway through the formation of the TAK1-TAB3-TRAF6 complex. These findings suggest TAB3 may be a useful prognostic biomarker in colorectal cancer and a target for treatment of metastatic colorectal cancer.

\section{INTRODUCTION}

Colorectal cancer (CRC) is one of the most common types of cancer and a leading cause of cancer-related deaths worldwide [1]. Metastasis and recurrence are the major causes of death in CRC. Although surgical resection is regarded as the standard curative treatment for CRC, the metastasis and recurrence rates after radical resection of CRC remain high [2]. Therefore, it is urgent to reveal the underlying molecular mechanisms involved in CRC progression and metastasis.

Survivin, a member of the inhibitor of apoptosis (IAP) protein family that inhibits caspases and blocks cell death, is highly expressed in most cancers and is associated with a poor clinical outcome $[3,4]$. Overexpression of Survivin has been reported in almost all human malignancies, including bladder cancer, lung cancer, breast cancer, stomach cancer, esophageal cancer, liver cancer, ovarian cancer and other cancer tissues [4-6]. Recently, emerging evidence has linked Survivin to tumor metastasis. For example, prior studies demonstrated that knockdown of Survivin in glioma inhibited angiogenesis [7], Survivin overexpression enhanced human melanocyte and melanoma cell migration [8] and Survivin promoted tumor cell invasion in vitro and metastatic dissemination in an in vivo murine model of breast cancer [9]. In CRC, Survivin overexpression is an independent poor prognostic factor in patients, and knockdown of Survivin could significantly inhibit CRC invasion and metastasis [10].These studies suggest that Survivin may play an important role in tumor invasion and metastasis.

In addition, previous studies have shown that Survivin is regulated by the NF- $\mathrm{KB}$ and PI3K/AKT signaling pathways in many cancers, such as bladder cancer, lymphoma and pancreatic cancer [11-14]. However, the mechanism by which Survivin is regulated in the process of CRC invasion and metastasis remains unclear. 
Transforming growth factor- $\beta$-activated kinase 1 (TAK1) is a member of the mitogen-activated protein kinase kinase kinase (MAPKKK) family, which is involved in signal transduction, inflammation and innate immune responses [15-17]. It is well established that TAK1 forms a complex with its binding proteins (TAB1, TAB2, and TAB3) and the TNF-receptor-associated factors (TRAFs), whereupon it stimulates $\mathrm{NF}-\kappa \mathrm{B}$ and mediates a wide range of biological processes $[18,19]$. TAB3, as a newly identified TAK1 binding partner, has been implicated in the immune response, signal transduction, inflammation and autophagy [20-22]. Recently, there has been an increasing number of studies on TAB3 in malignant tumors. TAB3 is markedly overexpressed in various tumor tissues, such as the testis, skin, non-small cell lung cancer (NSCLC), hepatocellular carcinoma (HCC) and small intestinal cancers [23-25]. In addition, a previous study reported that silencing of TAB3 inhibits NSCLC proliferation and chemoresistance via the $\mathrm{NF}-\kappa \mathrm{B}$ pathway [24]. In HCC, knockdown of TAB3 has been shown to enhance the rate of doxorubicin-induced apoptosis and chemosensitivity of HCC cells by acting

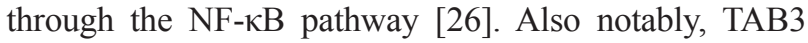
O-GlcNAcylation has been shown to promote breast cancer metastasis [27]. However, the role and specific mechanism of TAB3 in the process of CRC invasion and metastasis remain unclear.

In this study, we first demonstrated that TAB3 is upregulated in CRC tissues compared with non-tumor tissues and that the overexpression of TAB 3 is associated with metastasis and poor survival of CRC patients. Furthermore, functional studies provided the first evidence that TAB3 facilitates CRC invasion in vitro and metastasis in vivo by upregulating Survivin expression. We also demonstrated that TAB3 regulates Survivin through the $\mathrm{NF}-\kappa \mathrm{B}$ signaling pathway and activates NF- $\kappa \mathrm{B}$ through binding to the TAK1-TRAF6 complex.

\section{RESULTS}

\section{High TAB3 expression is associated with metastasis and poor overall survival in CRC}

To explore the expression and clinical significance of TAB3 in CRC, we first examined TAB3 expression in $68 \mathrm{CRC}$ tissue samples and corresponding adjacent normal tissues using western blotting. The western blotting results showed that TAB3 protein was markedly higher in $58.82 \%$ (40/68) of the CRC tissue samples than in their adjacent normal tissue (Figure 1A and 1B). We next examined TAB3 expression in $162 \mathrm{CRC}$ tissue samples and their paired adjacent normal tissues by immunohistochemistry (IHC). The IHC results showed that the TAB3 protein was highly expressed in $53.70 \%(87 / 162)$ of the CRC tissue samples and in only $8.64 \%(14 / 162)$ of the adjacent tissues (Figure 1C). These findings strongly indicate that TAB3 is overexpressed in CRC.
Next, we analyzed the association between TAB3 expression and clinicopathological parameters. The results revealed that high TAB3 expression was significantly associated with lymphatic metastasis $(P<0.001)$, venous invasion $(P=0.036)$ and advanced TNM stage $(P=0.001)$, indicating that TAB3 overexpression is involved in CRC aggressiveness and metastasis (Table 1). Furthermore, CRC patients in the high TAB3 expression group had a much shorter median survival time than those in the low TAB3 expression group (Figure 1D). Moreover, univariate and multivariate analyses using the Cox regression model revealed that the TAB3 level, lymphatic metastasis, venous invasion and TNM classification could be recognized as independent prognostic factors to evaluate the outcome of CRC patients (Supplementary Tables 1 and 2). This suggests that TAB3 potentially has clinical value as a predictive biomarker for disease outcome in CRC.

\section{Downregulation of TAB3 reduces Survivin expression and represses CRC invasion and metastasis in vitro and in vivo}

To investigate whether TAB3 regulated Survivin expression in CRC cells, we first examined the levels of TAB 3 and Survivin in various CRC cells by qRT-PCR and immunoblotting assays. Our results indicated that TAB3 was positively correlated with Survivin (Figure 2A). We then stably transfected the TAB3-specific short hairpin RNA (shTAB3\#1 and shTAB3\#2) into SW480 and LOVO cells to knockdown TAB3. qRT-PCR and western blotting results showed that the knockdown of TAB3 could decrease Survivin expression in the SW480 and LOVO cells $(P<0.01$, Figure 2B). Next, we observed that cell migration was significantly decreased in the shTAB3\#1 and shTAB3\#2 groups $(P<0.01$, Figure $2 \mathrm{C}$ and $2 \mathrm{D})$. Using a Matrigel-coated Transwell chamber, we found that the stable TAB3 knockdown SW480 and LOVO cells invaded through the matrix slower than that in the control group $(P<0.01$, Figure $2 \mathrm{E})$. Furthermore, an in vivo metastasis experiment further confirmed that the tumors formed by SW480-shTAB3\#1 cells showed decreased liver metastasis compared with the tumors formed by control cells (Figure $2 \mathrm{~F}$ and $2 \mathrm{G}$ ).

To verify the specificity of this tumor-promoting effect, we developed stable clones with TAB3 overexpression from HCT-116 and DLD-1 cells. The results showed that the overexpression of TAB3 led to marked upregulation of Survivin (Supplementary Figure 1A). The overexpression of TAB3 strongly enhanced in vitro $\mathrm{CRC}$ cell migration and invasion (Supplementary Figure 1B-1D). Consistent with the in vitro results, TAB3 overexpression increased liver metastasis in nude mice compared to control groups (Supplementary Figure 1E and 1F). Taken together, the results showed that the stable knockdown of TAB3 reduces Survivin expression and inhibit CRC invasion and metastasis. 


\section{Survivin is required for TAB3-mediated CRC invasion and metastasis in vitro and in vivo}

We utilized western blot and transwell assays to further investigate TAB3's regulation of Survivin and influence on CRC invasion and metastasis. The immunoblot analysis results showed that the knockdown of TAB3 decreased Survivin expression (Figure 3A and Supplementary Figure 2A), whereas the upregulation of Survivin attenuated the loss of Survivin expression in TAB3 knockdown SW480 and LOVO cells. We also found that the downregulation of TAB3 dramatically decreased the migration and invasion of SW480 and LOVO cells, whereas the upregulation of Survivin rescued the decreased migration and invasion abilities induced by TAB3 knockdown (Figure 3B and Supplementary Figure 2B). Furthermore, the in vivo assay also showed that the upregulation of Survivin rescued
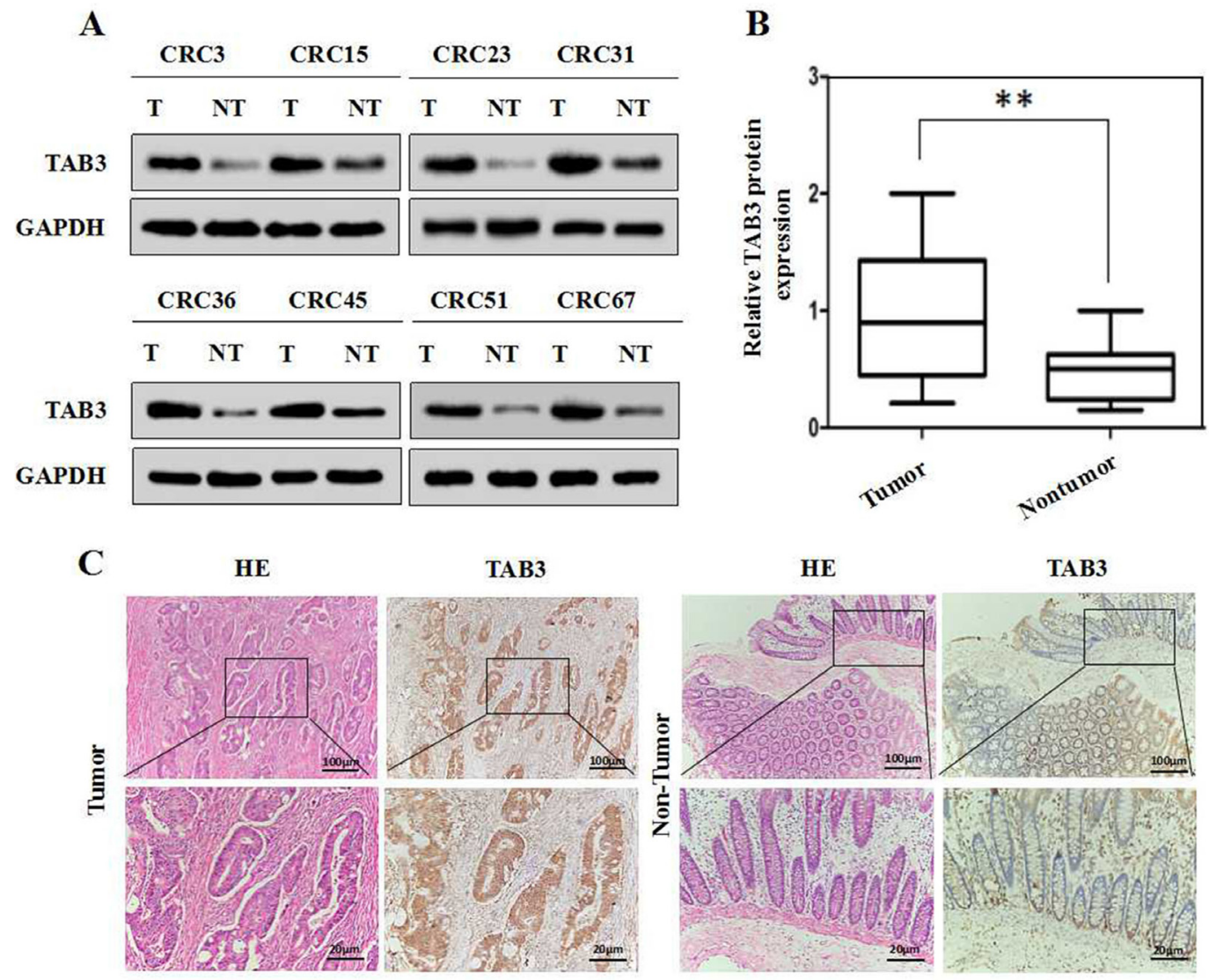

D
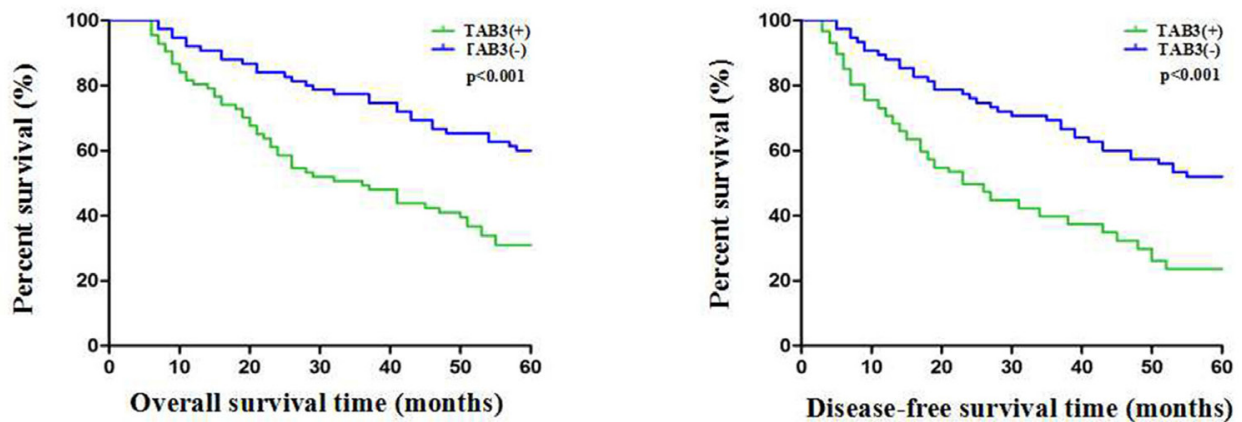

Figure 1: High TAB3 expression is associated with a poor prognosis in CRC patients. (A) Representative Western blot analysis of TAB3 protein expression (T: tumor, NT: non-tumor tissues). (B) Quantification of TAB3 protein expression using Western blot analyses in 68 paired CRC and their adjacent non-tumor tissues. GAPDH protein expression was used as an internal control $\left({ }^{* *} P<0.01\right)$. (C) IHC showed that TAB3 protein levels were increased in CRC tissues (magnification: 100×, inset magnification: 400×). (D) KaplanMeier survival curves for 162 CRC patients. Patients with high TAB3 protein expression levels showed significantly poorer overall survival and disease-free survival than patients with low TAB3 protein expression (both $P<0.001$, log-rank test). 
Table 1: Relationship between TAB3 expression and clinicopathological features in 162 CRC patients

\begin{tabular}{|c|c|c|c|c|}
\hline \multirow{2}{*}{ Parameters } & \multirow{2}{*}{$\frac{\text { Case }}{162}$} & \multicolumn{2}{|c|}{ TAB3 expression } & \multirow{2}{*}{$P$ value } \\
\hline & & Low & High & \\
\hline \multicolumn{5}{|l|}{ Age (years) } \\
\hline$<60$ & 102 & 51 & 51 & 0.255 \\
\hline$\geq 60$ & 60 & 24 & 36 & \\
\hline \multicolumn{5}{|l|}{ Gender } \\
\hline Female & 77 & 40 & 37 & 0.207 \\
\hline Male & 85 & 35 & 50 & \\
\hline \multicolumn{5}{|l|}{ Tumor size $(\mathrm{cm})$} \\
\hline$<5$ & 88 & 46 & 42 & 0.115 \\
\hline$\geq 5$ & 74 & 29 & 45 & \\
\hline \multicolumn{5}{|l|}{ Histological type } \\
\hline Differentiated & 130 & 65 & 65 & 0.075 \\
\hline Undifferentiated & 32 & 10 & 22 & \\
\hline \multicolumn{5}{|l|}{ Lymphatic metastasis } \\
\hline Negative & 91 & 58 & 33 & $<0.001^{* *}$ \\
\hline Positive & 71 & 17 & 54 & \\
\hline \multicolumn{5}{|l|}{ Venous invasion } \\
\hline Negative & 116 & 60 & 56 & $0.036^{*}$ \\
\hline Positive & 46 & 15 & 31 & \\
\hline \multicolumn{5}{|l|}{ TNM staging } \\
\hline $\mathrm{I} / \mathrm{II}$ & 97 & 55 & 42 & $0.001^{* *}$ \\
\hline III/IV & 65 & 20 & 45 & \\
\hline
\end{tabular}

$P$ value was generated by comparing all subgroups and analyzed by the chi-square test. $P<0.05$ was considered as statistically significant.

the decreased incidence of liver metastasis of the SW480shTAB3\#1 group (Figure 3C and 3D).

Next, we decreased Survivin expression in TAB3overexpressing CRC cells and then measured TAB3 and Survivin protein levels and cell migration and invasion. Immunoblotting showed that the overexpression of TAB3 significantly increased Survivin expression, whereas Survivin knockdown dramatically inhibited the increase in Survivin expression induced by TAB3 in HCT116 and DLD-1 cells (Figure 3E and Supplementary Figure 2C). Meanwhile, the downregulation of Survivin significantly reduced TAB3-enhanced cell migration and invasion (Figure 3F and Supplementary Figure 2D). In vivo results showed that downregulation of Survivin decreased the incidence of liver metastasis of the HCT116-pcDNA3.1(+)-TAB3 group (Figure $3 \mathrm{G}$ and $3 \mathrm{H}$ ). Thus, these results demonstrate that Survivin is essential for TAB3-mediated CRC metastasis.

\section{TAB3 regulates Survivin expression through the NF- $K B$ pathway in CRC cells}

To further clarify the mechanism by which TAB3 regulates Survivin in CRC cells, we hypothesized that TAB3 regulates Survivin via the NF- $\mathrm{\kappa B}$ signaling in CRC cells. We first measured the changes in phosphorylatedI $\kappa \alpha(p-I \kappa B \alpha)$, phosphorylated-p65 (p-p65), total p65 expression and NF- $\mathrm{kB}$ activity in TAB3 knockdown SW480 and LOVO cells. The results showed that the knockdown of TAB3 can significantly decrease $\mathrm{p}-\mathrm{I} \kappa \mathrm{B} \alpha, \mathrm{p}-\mathrm{p} 65$ and the activity of NF- $\mathrm{BB}$ luciferase reporter in SW480 and LOVO cells (Figure 4A, 4B and Supplementary Figure 3A, 3B). We also found that the knockdown of TAB3 decreased other downstream genes in the NF- $\mathrm{kB}$ pathway, including c-Myc and MMP-9 in SW480 and LOVO cells. Whereas, the activation of $\mathrm{NF}-\kappa \mathrm{B}$ signaling by treatment with $\mathrm{TNF} \alpha(10 \mathrm{ng} / \mathrm{ml})$ 
attenuated the loss of this protein expression in TAB3knockdown SW480 and LOVO cells (Figure 4C and Supplementary Figure $3 \mathrm{C}$ ). In addition, we found that the activation of NF- $\kappa \mathrm{B}$ signaling rescued the decreased cell migration and invasion induced by the knockdown of TAB3 (Figure 4D and Supplementary Figure 3D).

To further verify that TAB3 regulates Survivin expression through the NF- $\mathrm{B}$ pathway, we investigated the effect of TAB3 overexpression on the NF- $\kappa$ B pathway. The results showed that the upregulation of TAB3 expression significantly increased $\mathrm{p}-\mathrm{I} \kappa \mathrm{B} \alpha, \mathrm{p}-\mathrm{p} 65$ and the activity of NF- $\kappa$ B luciferase reporter (Figure $4 \mathrm{E}-4 \mathrm{~F}$ and Supplementary Figure 3E-3F). We then detected the expression of Survivin, c-Myc and MMP-9 in HCT116- and DLD-1-pcDNA3.1(+)-TAB3 cells treated with the NF- $\kappa$ B inhibitor caffeic acid phenethyl ester (CAPE, $10 \mathrm{ng} / \mathrm{ml}$ ). The results showed that the overexpression of TAB3 increased the expression of Survivin, c-Myc and MMP-9 proteins. Whereas, blockade of NF- $\mathrm{BB}$ signaling dramatically inhibited the increase of Survivin, c-Myc and MMP-9 expression in HCT-116- and DLD-1-
pcDNA3.1(+)-TAB3 cells (Figure 4G and Supplementary Figure $3 \mathrm{G}$ ). Meanwhile, the transwell assay showed that blockade of NF- $\mathrm{KB}$ signaling dramatically decreased TAB3-induced cell migration and invasion (Figure $4 \mathrm{H}$ and Supplementary Figure $4 \mathrm{H}$ ). These studies confirmed that TAB3 regulates Survivin-induced CRC migration and invasion through the NF- $\mathrm{BB}$ pathway.

\section{TAB3 activates NF- $\mathrm{KB}$ by binding to the TAK1- TRAF6 complex in CRC cells}

Previous studies have shown that TAB2, TRAF6 and TAK1 are involved in NF- $\mathrm{BB}$ activation [28, 29]. To determine whether the TAK1-TAB3-TRAF6 complex could also activate the NF- $\kappa \mathrm{B}$ pathway in CRC cells, we first observed whether TAB3, TRAF6 and TAK1 directly interacted in CRC cells. The Co-IP study showed that endogenous TAB3, TRAF6 and TAK1 were detected in the immunoprecipitant, demonstrating the interaction among TAB3, TRAF6 and TAK1 (Figure 5A-5C and Supplementary Figure 4A-4C).
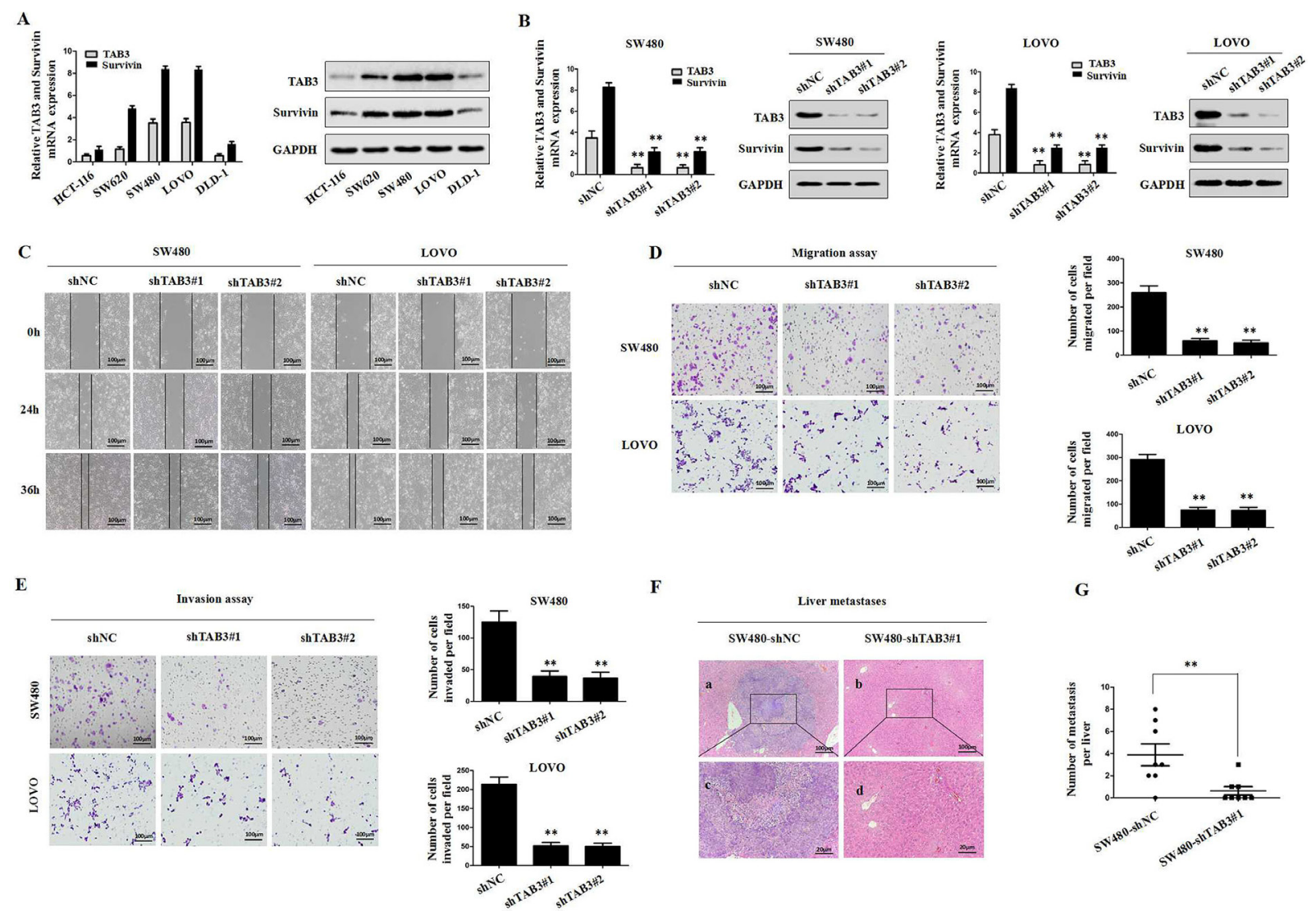

F

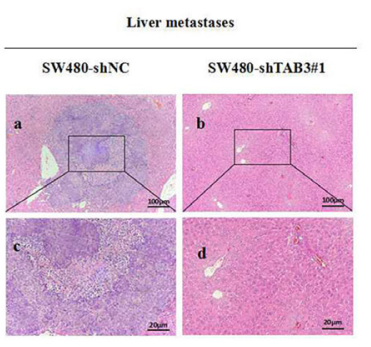

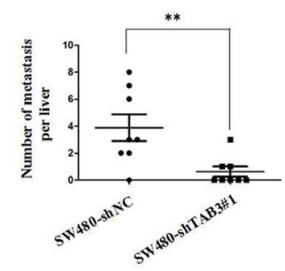

Figure 2: Stable knockdown of TAB3 reduced Survivin expression and inhibited CRC invasion and metastasis in vitro and in vivo. (A) qRT-PCR and Western blot analyses of TAB3 and Survivin expression in the indicated CRC cell lines. (B) Western blot analyses were used to detect TAB3 and Survivin expression in SW480 and LOVO cells stably transfected with shNC, shTAB3\#1 and shTAB3\#2. (C) Wound healing assay. Wound closure was delayed in stable TAB3-knockdown cells compared with the shNC control at both the 24- and 36-h time points. Magnification, $\times 100$. (D) Transwell migration assays of SW480 and LOVO cells with TAB3 expression stably inhibited $\left.{ }^{* *} p<0.01\right)$. Magnification, $\times 100$. (E) Transwell invasion assays of SW480 and LOVO cells treated with shNC, shTAB3\#1 and shTAB3\#2. Magnification, $\times 100$. (F) Representative H\&E staining of livers from the SW480-shNC and SW480-shTAB3 \#1 groups. Magnification: $\mathrm{a}$ and $\mathrm{b}, \times 100 ; \mathrm{c}$ and $\mathrm{d}, \times 400$. (G) $\left(n=8 ;{ }^{* *} P<0.01\right)$. 

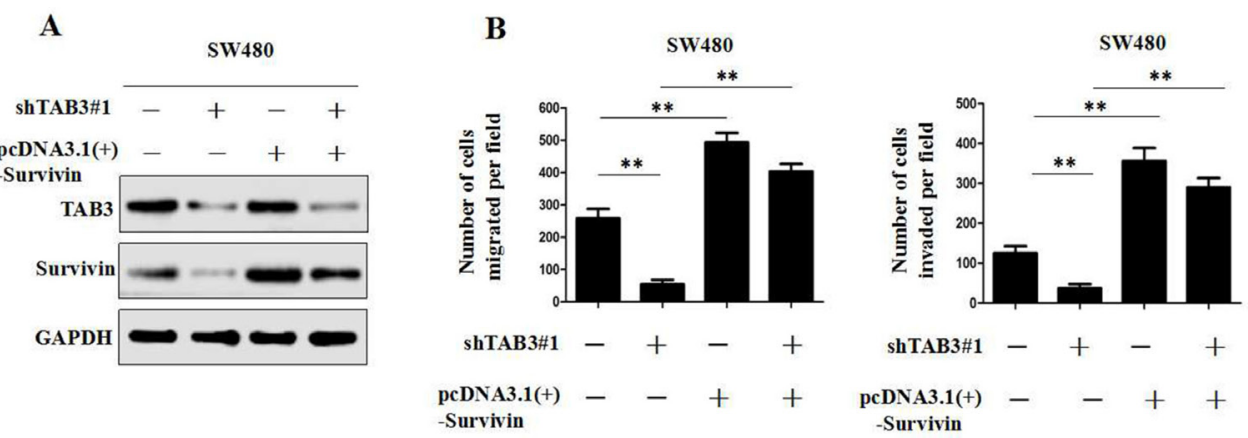

C

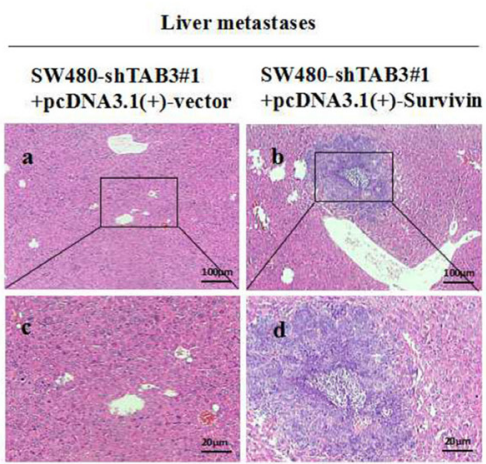

D
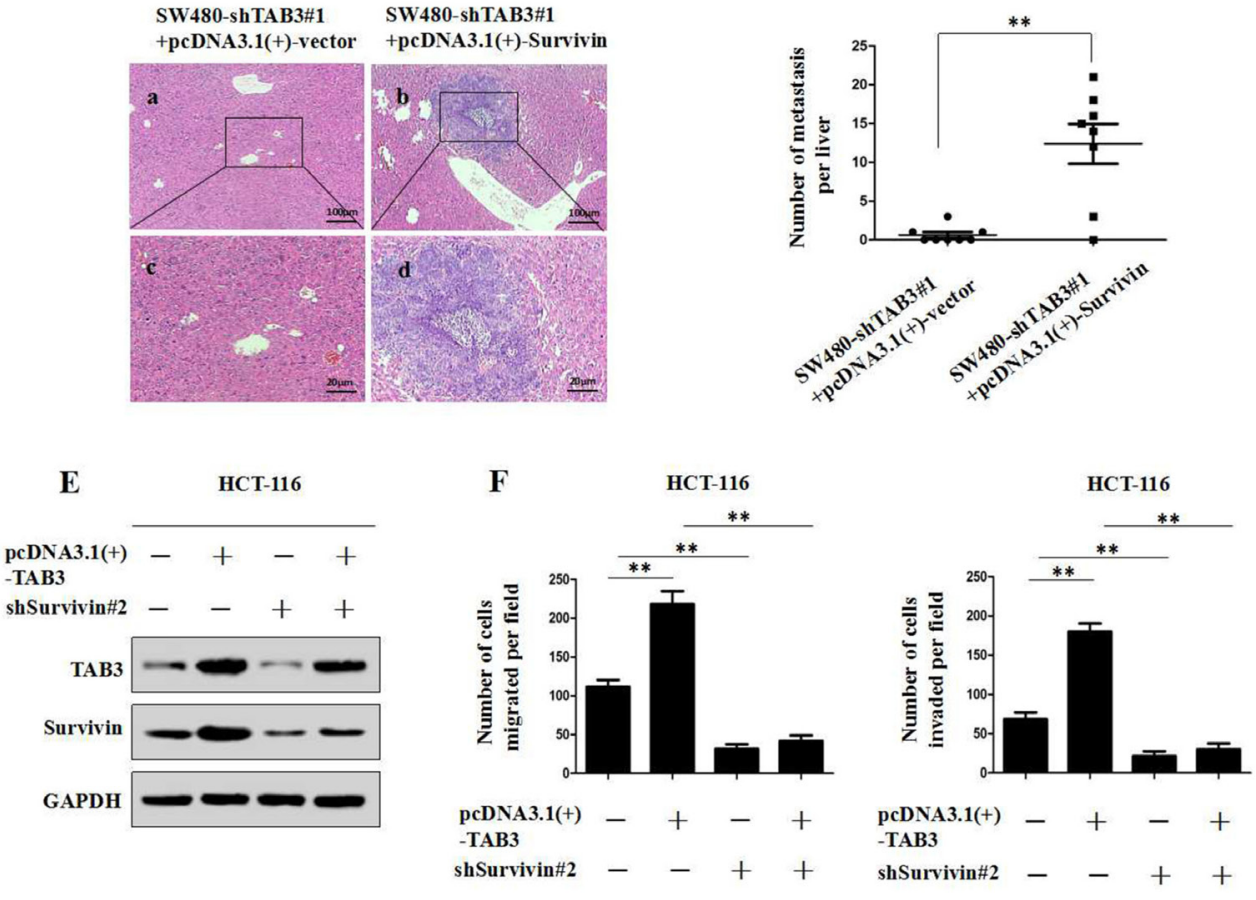

G

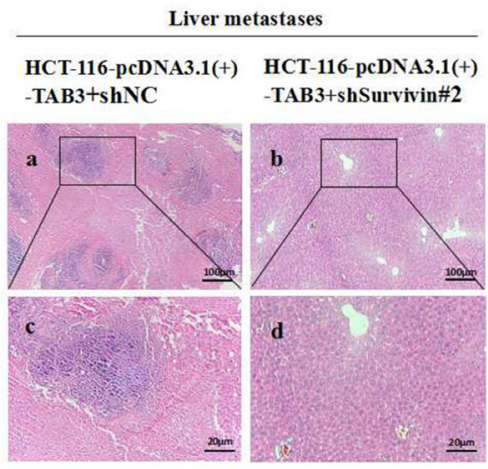

$\mathbf{H}$

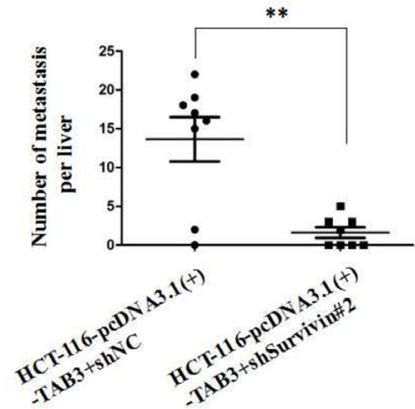

Figure 3: TAB3 promotes CRC invasion and metastasis by upregulating Survivin expression. (A) The upregulation of Survivin attenuated the loss of Survivin expression in SW480-shTAB3 cells. (B) Transwell assays showed that the upregulation of Survivin significantly rescued cell migration and invasion in SW480-shTAB3 cells $\left({ }^{* *} P<0.01\right)$. (C) Representative H\&E staining of livers from the SW480-shTAB3\#1 +pcDNA3.1(+)-vector and SW480-shTAB3\#1 +pcDNA3.1(+)-Survivin groups is shown. Magnification: a and b, $\times 100$; $\mathrm{c}$ and d, $\times 400$. (D) $\left(n=8 ;{ }^{* *} P<0.01\right)$. (E) Protein levels of TAB3 and Survivin were detected by Western blot analysis. The knockdown of Survivin expression dramatically inhibited the increase of Survivin expression in HCT116-TAB3 cells. (F) Transwell assays showed that Survivin inhibition reduced TAB3-enhanced cell migration and invasion $\left.{ }^{* *} P<0.01\right)$. (G) Representative H\&E staining of livers from the HCT116-TAB3+shNC and HCT116-TAB3+shSurvivin\#2 groups is shown. Magnification: a and b, $\times 100$; $\mathrm{c}$ and $\mathrm{d}, \times 400$. (H) $\left(n=8 ;{ }^{* *} P<0.01\right)$. 
In addition, we transfected shTAB3, shTRAF6 or shTAK1 plasmids into SW480 and LOVO cells and evaluated the effects of variable TAB3, TRAF6 and TAK1 complex on the NF- $\kappa \mathrm{B}$ pathway. The results showed that the knockdown of TAB3, TRAF6 or TAK1 decreased the interaction among TAB3, TRAF6 and TAK1, and significantly decreased p-IKB $\alpha$ and p-p65 levels (Figure 5D-5F and Supplementary Figure 4D-4F). Whereas, the overexpression of TAB3, TRAF6 or TAK1 increased the TAB3, TRAF6 and TAK1 complex, as well as p-IKB $\alpha$ and p-p65 levels (Figure 5G-5I and Supplementary Figure 4G-4I). These data demonstrate that the TAK1-TAB3-TRAF6 complex is involved in NF$\mathrm{kB}$ activation.

To further determine whether TAB3 activates NF- $\kappa B$ through the formation of the TAK1-TAB3TRAF6 complex, we measured the changes in $\mathrm{p}-\mathrm{IKB} \alpha$ and p-p65 levels and NF- $\kappa B$ transcriptional activity in the synchronous TAB3, TAK1 and TRAF6 knockdown SW480 and LOVO cells. The results showed that reducing TAB3, TAK1 or TRAF6 expression could significantly inhibit p-IKB $\alpha$ and p-p65 levels and NF- $\kappa \mathrm{B}$ transcriptional activity. Synchronous knockdown of TAB3, TAK1 and TRAF6 showed the strongest suppression effect (Figure 5J-5K and Supplementary Figure 4J-4K). Next, we investigated the effect of TAB3, TAK1 and TRAF6 synchronous overexpression on the NF- $\mathrm{B}$ pathway. The results showed that the overexpression of TAB3, TAK1 or TRAF6 increased p-I $\kappa \mathrm{B} \alpha$ and p-p65 levels and $\mathrm{NF}-\kappa \mathrm{B}$ transcriptional activity, and the synchronous overexpression of TAB3, TAK1 and TRAF6 showed the strongest enhancement effect (Figure 5L-5M and Supplementary Figure 4L-4M). These data demonstrate that TAB3 activates NF- $\kappa$ B through binding to the TAK1TRAF6 complex.

\section{DISCUSSION}

Colorectal cancer (CRC) is one of the most common malignant cancers, and approximately $50-60 \%$ of patients present with metastases at the initial diagnosis [30, 31]. Tumor metastasis is a complex, multi-stage process that
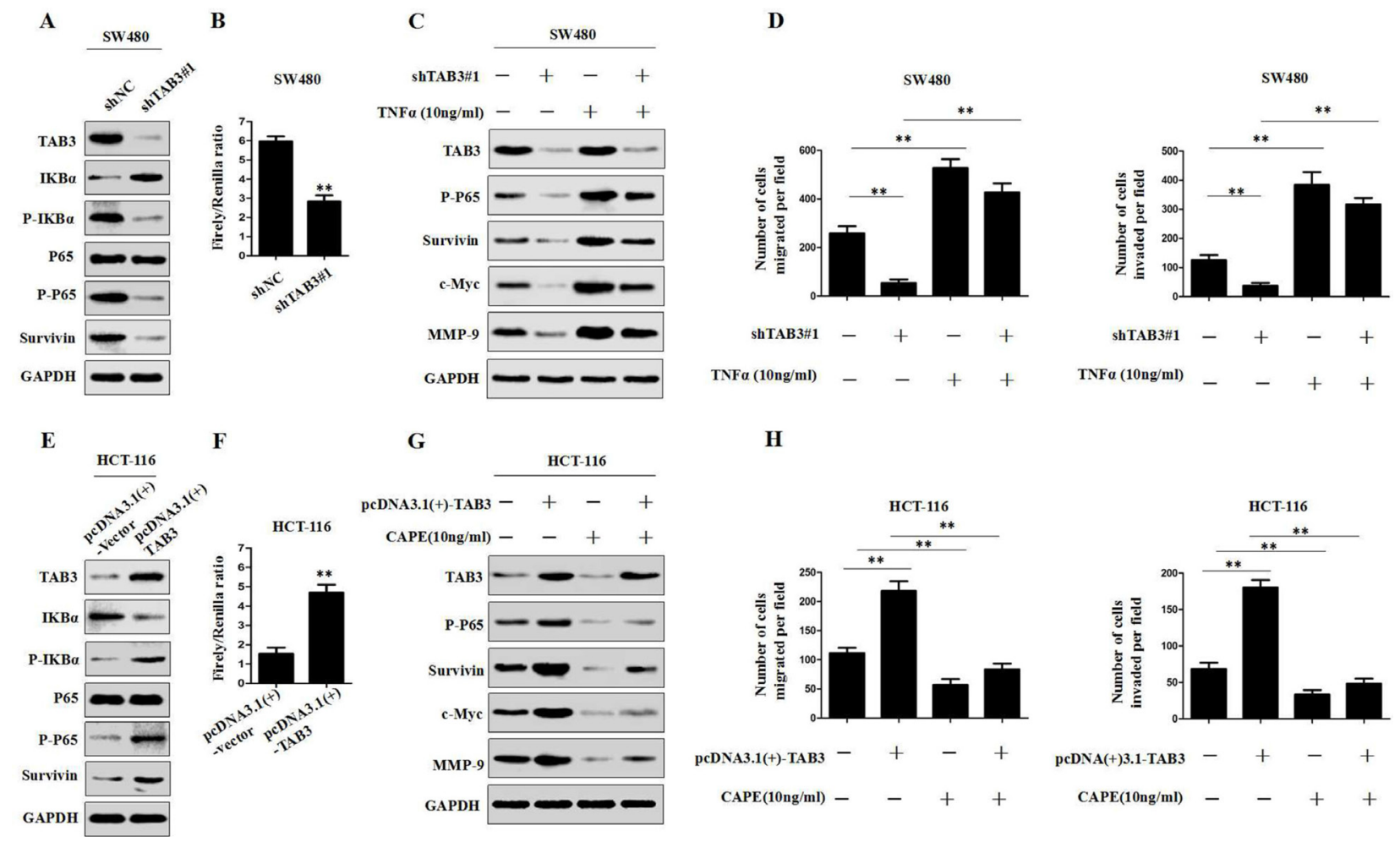

$\mathbf{H}$

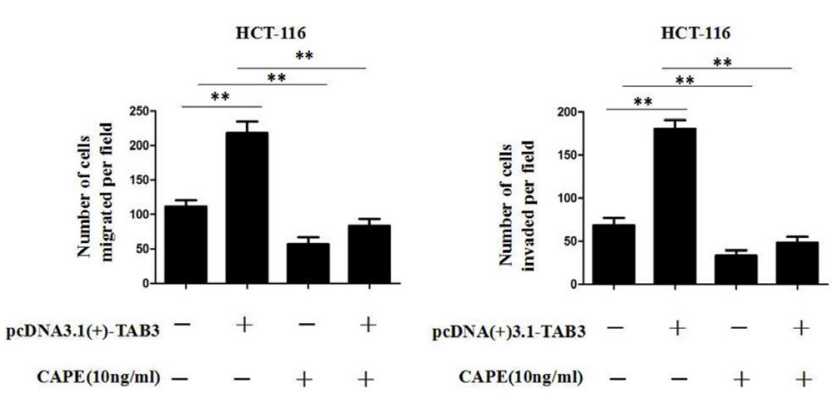

Figure 4: TAB3 regulates Survivin expression through the NF-kB pathway. (A) The protein expression level of TAB3, IKBa, p-IKBa, P65, p-P65 and Survivin were assessed by Western blotting in TAB3-silenced SW480 cells. (B) Luciferase analysis was used to determine the NF- $\mathrm{KB}$ activity in TAB3-knockdown SW480 cells ( $\left.{ }^{* *} P<0.01\right)$. (C) Western blot analysis showing the levels of $\mathrm{TAB} 3$ silencing and activation of the NF- $\mathrm{KB}$ pathway (treatment with $10 \mathrm{ng} / \mathrm{ml}$ tumor necrosis factor) and their effects on $\mathrm{p}-\mathrm{P} 65$, Survivin, c-Myc, and MMP-9 in SW480 cells. (D) Activation of the NF- $\mathrm{BB}$ pathway rescued cell migration and invasion in SW480-shTAB3 cells $\left({ }^{* *} P<0.01\right)$. (E) Protein expression levels of TAB3, IKBa, p-IKBa, P65, p-P65 and Survivin in HCT116 cells transfected with pcDNA3.1(+)vector and pcDNA3.1(+)-TAB3. (F) Luciferase analysis was performed using HCT116 cells transfected with the pcDNA3.1(+)-vector or pcDNA3.1(+)-TAB3 plasmid $\left({ }^{* *} P<0.01\right)$. (G) Western blot analysis showing the levels of TAB3 overexpression and NF- $\mathrm{KB}$ signaling inhibition (treatment with $10 \mathrm{ng} / \mathrm{ml}$ Caffeic Acid Phenethyl Ester) and their effects on p-P65, Survivin, c-Myc and MMP-9 in HCT116 cells.(H.) Blockade of the NF- $\kappa$ B pathway enhanced migration and invasion in HCT116-pcDNA3.1 $(+)$-TAB3 cells $\left({ }^{* *} P<0.01\right)$. 
is classically simplified as local invasion, intravasation, survival in circulation, extravasation and colonization $[32,33]$. The overexpression of metastasis initiation genes and their targets often predicts a poor prognosis in various types of cancer. Understanding the molecular mechanisms underlying $\mathrm{CRC}$ metastasis is of crucial significance to the development of therapeutic strategies for advanced CRC patients.

Transforming growth factor- $\beta$-activated kinase 1 (TAK1)-binding protein 3 (TAB3) belongs to a family of TABs that play an essential role in immunity [20]. Recent emerging evidence has indicated that TAB3 also plays a crucial role in cancer development and chemoresistance. For instance, the previously study confirmed that TAB3 is a downstream effector of TGF $\beta$ pathway [18], and TGF $\beta$ pathway has been demonstrated to be involved in the chemoresistance of CRC [34, 35]. Another study found that TAB3 was specifically expressed in triple-negative breast cancer, and it was significantly correlated with a poor prognosis in the patients [27]. However, the impact of TAB3 on CRC invasion and metastasis remains unclear.

In this study, we observed that TAB3 expression was significantly upregulated in CRC tissues and was associated with a poor prognosis in CRC patients. We also demonstrated that TAB3 promoted CRC cell invasion and metastasis both in vitro and in vivo and investigated the mechanism by which TAB3 affects invasion and metastasis in CRC cells.

Survivin, a member of the inhibitor of apoptosis family proteins [3], is highly expressed in CRC and associated with a poor clinical outcome [36]. In this study, we identified TAB3 as a novel regulator of Survivin. Our data indicate that TAB3 inhibition can reduce Survivin expression and decrease the invasion and metastasis of
$\mathbf{A}$

B
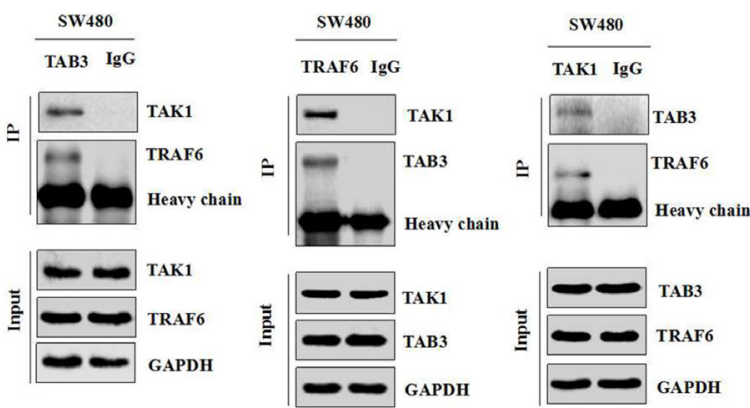

G

G $\quad$ HCT-116

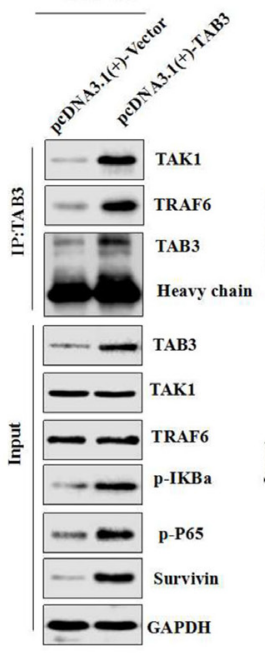

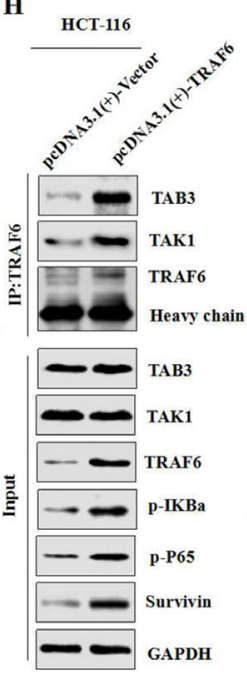

H
I $\quad$ HCT-116

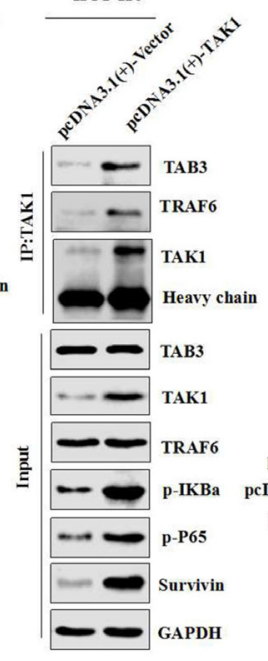

D

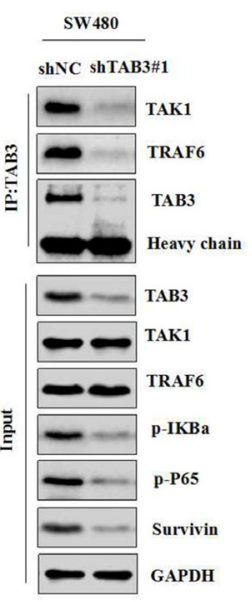

$\mathbf{J}$

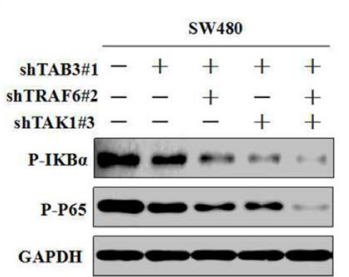

$\mathbf{L}$

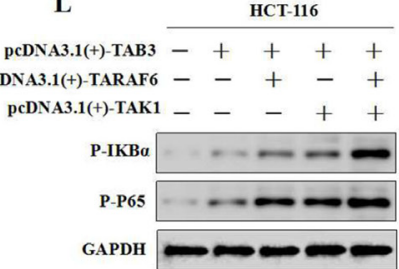

$\mathbf{E}$

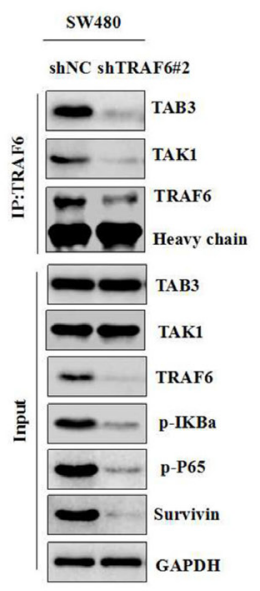

$\mathbf{F}$

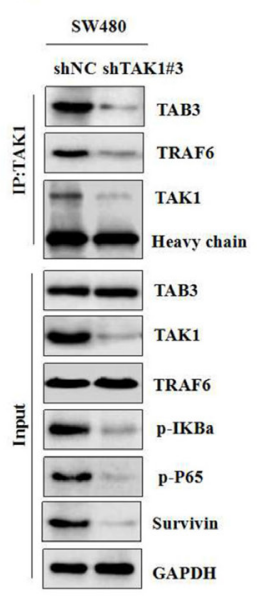

$\mathbf{K}$

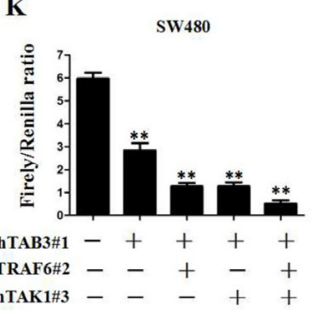

M

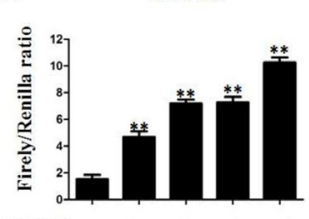

pCDNA3.1(+)-TAB3 -++++ pcDNA3.1(+)-TARAF6 - -+-+

Figure 5: TAB3, TRAF6 and TAK1 are involved in NF-кB activation. (A-C) co-IP among endogenous TAB3, TAK1 and TRAF6 in SW480 cells. (D-I) co-IP and Western blot analysis showing the levels of TAB3, TAK1 and TRAF6 after silencing or overexpression and their effects on TAB3, TAK1 and TRAF6 interaction and the NF- $\kappa$ B pathway in CRC cells. (J-K) Western blot and Luciferase analysis showing the levels of TAB3, TAK1 and TRAF6 after silencing and their effects on p-IKBa and p-P65 expression and NF- $\kappa$ B activity in SW480 cells $\left({ }^{* *} P<0.01\right)$. $(\mathbf{L}-\mathbf{M})$ Western blot and Luciferase analysis showing the levels of TAB3, TAK1 and TRAF6 after overexpression and their effects on p-IKBa and p-P65 expression and NF- $\kappa$ B activity in HCT-116 cells $\left({ }^{* *} P<0.01\right)$. 
CRC in vitro and in vivo. Moreover, the upregulation of Survivin rescued the decreased invasion and liver metastasis induced by TAB3 knockdown. Whereas, inhibition of Survivin significantly decreased TAB3induced invasiveness and metastasis. These results suggest that one of the mechanisms by which TAB3 promotes CRC metastasis involves the upregulation of Survivin expression.

Furthermore, the mechanism by which TAB3 regulates Survivin was further investigated. Our investigation focused on the NF- $\kappa \mathrm{B}$ signaling pathway. We determined that the knockdown of TAB 3 decreased $\mathrm{p}-\mathrm{I} \kappa \mathrm{B} \alpha$ and $\mathrm{p}-\mathrm{p} 65$ levels and NF- $\kappa \mathrm{B}$ activity. This ultimately lead to the downregulation of its target genes, such as c-Myc and MMP-9, whereas activation of NF- $\kappa$ B signaling rescued the decreased expression of Survivin and other NF- $\kappa$ B signaling components induced by TAB3 knockdown. Furthermore, the transwell assay indicated that the activation of NF- $\kappa \mathrm{B}$ signaling significantly rescued the decreased cell migration and invasion induced by the knockdown of TAB3. Taken together, these studies demonstrated that TAB3 regulates Survivin-mediated $\mathrm{CRC}$ migration and invasion through the NF- $\mathrm{B}$ pathway.

In addition, we further investigated the mechanism by which TAB 3 regulates the NF- $\kappa \mathrm{B}$ pathway. Previous studies confirmed that TAB2 acts as an adaptor that links TAK1 and TRAF6 to mediate NF- $\mathrm{B}$ [28]. Comparative bioinformatics revealed that TAB3 is similar to TAB2 in that they both contain 3 putative functional domains with the same relative organization [29]. Such striking similarities lead us to hypothesize that the TAK1-TAB3TRAF6 complex could also activate the NF- $\kappa$ B pathway in CRC cells. In this study, we demonstrated that TAB3, TRAF6 and TAK1 directly interacted in CRC cells and found that the TAB3, TRAF6 and TAK1 complex are involved in NF- $\kappa \mathrm{B}$ activation in CRC cells. We also found that TAB3 activates NF- $\kappa \mathrm{B}$ through the formation of the TAK1-TAB3-TRAF6 complex.

In summary, we demonstrated that TAB3 promotes CRC metastasis by regulating Survivin expression through the NF- $\kappa \mathrm{B}$ signaling pathway by binding to the TAK1TRAF6 complex. The newly identified TAB3-NF- $\kappa$ BSurvivin axis provides new insight into the underlying mechanisms of CRC invasion and metastasis, evidence of a valuable prognostic biomarker in CRC and a rationale for treatment of metastatic CRC.

\section{MATERIALS AND METHODS}

\section{Patients and samples}

Human CRC specimens were collected from 162 patients who underwent CRC resection at the Second Affiliated Hospital of Nanchang University and Jiangxi Provincial Cancer Hospital between January 2008 and December 2011. The study protocol was approved by the Ethics Committee of the Second Affiliated Hospital of Nanchang University and Jiangxi Provincial Cancer Hospital.

\section{Cell culture, plasmids and reagents}

The human CRC cell lines SW480, SW620, LOVO, DLD-1 and HCT-116 were purchased from the Shanghai Institute of Cell Biology. The cells were cultured in RPMI 1640 medium (Gibco, Grand Island, NY, USA) supplemented with fetal bovine serum (HyClone, Logan, UT, USA) to a final concentration of $10 \%$ and antibiotics at $37^{\circ} \mathrm{C}$ with $5 \% \mathrm{CO}_{2}$. Plasmids and reagents are described in the Supplementary Materials and Methods. All these cells were authenticated using short tandem repeat profiling by the Cell Bank.

\section{Immunohistochemistry}

The CRC and adjacent tissues were treated with xylene, graded alcohol and then antigen retrieval was performed in $0.01 \mathrm{M}$ citrate buffer. Hydrogen peroxide was used for blockage. Tissue sections were treated with goat serum for 30 minutes. After that, the slides was incubated anti-TAB3 polyclonal antibodies (Abcam, Cambridge, MA, USA, 1:300 dilution) overnight at $4^{\circ} \mathrm{C}$. A 2-step immunohistochemical method (catalog:PV-9000; ZSGBBIO Co., Ltd., Beijing, China) was adopted to perform the immunostaining. The staining intensity and percentage of positive cells were scored semi-quantitatively by 3 pathologists who were blind to the clinical parameters.

\section{qRT-PCR, H\&E, western blot and co- immunoprecipitation (Co-IP) analyses}

All qRT-PCR, western blot, and Co-IP procedures were performed as previously described [37]. The specific primers used for PCR amplification are shown in Supplementary Table 3.

\section{Wound-healing assay}

The cells were grown to $80-90 \%$ confluence in $60 \mathrm{~mm}$ dishes. Artificial wounds were generated by scraping a pipette tip across the cell surface. After the removal of the detached cells by gentle washing with PBS, the cells were fed with fresh complete medium and were incubated over time to allow the cells to migrate into the open area. Cell movement during wound closure was measured by phase-contrast photography at $37^{\circ} \mathrm{C}$ for incubations of 0,24 and $36 \mathrm{hrs}$, and 3 randomly selected wound areas were analyzed.

\section{Cell migration and invasion assay}

For the migration assay, $5 \times 10^{4}$ cells were resuspended in serum-free medium and were placed in the upper 
chambers. For the invasion assays, $1 \times 10^{5}$ cells were seeded in a Matrigel-coated chamber (BD Biosciences, Bedford, MA, USA). After $24 \mathrm{hrs}$ (to examine migration) or $48 \mathrm{hrs}$ (to examine invasion) of incubation, the non-migrated cells on the upper surface of the membrane were removed, and the cells on the lower surface were fixed and stained with $0.1 \%$ crystal violet. The cells in 5 random microscopic fields were counted and imaged using a light microscope with a DP70 CCD system (Olympus Corp., Tokyo, Japan).

\section{Luciferase reporter assay}

For the NF- $\kappa \mathrm{B}$ transcriptional activity assay, $500 \mathrm{ng}$ of PGL3.0-NF-kB-luc and $50 \mathrm{ng}$ of Renilla luciferase plasmids or $500 \mathrm{ng}$ of PGL3.0-luc and $50 \mathrm{ng}$ of Renilla luciferase plasmids were co-transfected into TAB3overexpression or TAB3-knockdown CRC cells. After $48 \mathrm{hrs}$ of treatment, Luciferase and Renilla signals were measured using a Dual Luciferase Reporter Assay kit (Promega) according to the manufacturer's protocol.

\section{In vivo metastasis assay}

For in vivo metastasis assays, $1 \times 10^{7}$ cells in $100 \mathrm{ml}$ phosphate-buffered saline were inoculated subcutaneously onto the dorsal surfaces of $\mathrm{BALB} / \mathrm{c}$ nude male mice (Shanghai SLAC Laboratory Animal Co., Ltd., China). Once xenografts were established, they were excised and minced into $1 \mathrm{~mm}^{3}$ pieces. One of these pieces was then orthotopically implanted at the ileocecal junction of another $\mathrm{BALB} / \mathrm{c}$ nude mouse (eight in each group, male BALB/cnu/nu, 6-8 weeks). The mice were sacrificed 35 days after tumor implantation [38]. The animal study was approved by the Ethics Committee for Animal Experiments of the Second Affiliated Hospital of Nanchang University and was performed in accordance with the "Guide for the Care and Use of Laboratory Animals” (revised 1985).

\section{Statistical analysis}

All data were analyzed using SPSS (Statistical Package for the Social Sciences) 19.0 (SPSS, Inc.). The patient survival curve was calculated using the KaplanMeier method. Univariate and multivariate Cox proportional hazards regression analyses were performed to estimate the crude hazard ratios (HRs), adjusted HRs and 95\% confidence intervals (CIs) of HRs. Differences between groups were analyzed using Student's $t$-test when comparing two groups or one-way analysis of variance (ANOVA) when comparing more than 2 groups. Differences were considered statistically significant at $P<0.05$.

\section{Abbreviations}

TAK1, Transforming growth factor- $\beta$-activated kinase 1; TABs, TAK1-binding proteins; TRAFs, TNF- receptor-associated factors; $\mathrm{CRC}$, colorectal cancer; IHC, immunohistochemistry; qRT-PCR, real-time quantitative polymerase chain reaction; Co-IP, co-immunoprecipitation; NSCLC, non-small cell lung cancer.

\section{CONFLICTS OF INTEREST}

There are no conflicts of interest to disclose.

\section{FUNDING}

This study was supported by grants from the National Natural Science Foundation of China (Nos. 81560396,81560475 and 81360325), the Project of Jiangxi Provincial Department of Science and Technology (Nos. 20171BAB205060 and 20171BAB215024), the Project of Jiangxi Provincial Department of Education (Nos. GJJ150142 and GJJ160251), and the advantage innovation team of Jiangxi Province (No. 20153BCB24004).

\section{REFERENCES}

1. Torre LA, Bray F, Siegel RL, Ferlay J, Lortet-Tieulent J, Jemal A. Global cancer statistics, 2012. CA Cancer J Clin. 2015; 65:87-108.

2. De Rosa M, Pace U, Rega D, Costabile V, Duraturo F, Izzo P, Delrio P. Genetics, diagnosis and management of colorectal cancer (Review). Oncol Rep. 2015; 34:1087-1096.

3. Tamm I, Wang Y, Sausville E, Scudiero DA, Vigna N, Oltersdorf T, Reed JC. IAP-family protein Survivin inhibits caspase activity and apoptosis induced by Fas (CD95), Bax, caspases, and anticancer drugs. Cancer Res. 1998; 58:5315-5320.

4. Chen X, Duan N, Zhang C, Zhang W. Survivin, Tumorigenesis: Molecular Mechanisms and Therapeutic Strategies. J Cancer. 2016; 7:314-323.

5. Dong D, Jiang W, Lei J, Chen L, Liu X, Ge J, Che B, Xi X, Shao J. Ubiquitin-like protein FAT10 promotes bladder cancer progression by stabilizing survivin. Oncotarget. 2016; 7:81463-81473. https://doi.org/10.18632/oncotarget. 12976.

6. Li S, Wang L, Meng Y, Chang Y, Xu J, Zhang Q. Increased levels of LAPTM4B, VEGF and survivin are correlated with tumor progression and poor prognosis in breast cancer patients. Oncotarget. 2017; 8:41282-41293. https://doi. org/10.18632/oncotarget.17176.

7. Wang P, Zhen H, Zhang J, Zhang W, Zhang R, Cheng X, Guo G, Mao X, Wang J, Zhang X. Survivin promotes glioma angiogenesis through vascular endothelial growth factor and basic fibroblast growth factor in vitro and in vivo. Mol Carcinog. 2012; 51:586-595.

8. McKenzie JA, Liu T, Goodson AG, Grossman D. Survivin enhances motility of melanoma cells by supporting Akt 
activation and \{alpha\} 5 integrin upregulation. Cancer Res. 2010; 70:7927-7937.

9. Liu S, Huang W, Jin MJ, Fan B, Xia GM, Gao ZG. Inhibition of murine breast cancer growth and metastasis by Survivin-targeted siRNA using disulfide cross-linked linear PEI. Eur J Pharm Sci. 2016; 82:171-182.

10. Chu XY, Chen LB, Wang JH, Su QS, Yang JR, Lin Y, Xue LJ, Liu XB, Mo XB. Overexpression of Survivin is correlated with increased invasion and metastasis of colorectal cancer. J Surg Oncol. 2012; 105:520-528.

11. Cui X, Shen D, Kong C, Zhang Z, Zeng Y, Lin X, Liu $\mathrm{X}$. NF-kappaB suppresses apoptosis and promotes bladder cancer cell proliferation by upregulating Survivin expression in vitro and in vivo. Sci Rep. 2017; 7:40723.

12. Wu M, Chen J, Wang Y, Hu J, Liu C, Feng C, Zeng X. URGCP/URG4 promotes apoptotic resistance in bladder cancer cells by activating NF-kappaB signaling. Oncotarget. 2015; 6:30887-30901. https://doi.org/10.18632/oncotarget. 5134.

13. Sun L, Zhao Y, Shi H, Ma C, Wei L. LMP-1 induces Survivin expression to inhibit cell apoptosis through the NF-kappaB and PI3K/Akt signaling pathways in nasal NK/T-cell lymphoma. Oncol Rep. 2015; 33:2253-2260.

14. Yi XP, Han T, Li YX, Long XY, Li WZ. Simultaneous silencing of XIAP and Survivin causes partial mesenchymal-epithelial transition of human pancreatic cancer cells via the PTEN/PI3K/Akt pathway. Mol Med Rep. 2015; 12:601-608.

15. Shibuya H, Iwata H, Masuyama N, Gotoh Y, Yamaguchi K, Irie K, Matsumoto K, Nishida E, Ueno N. Role of TAK1 and TAB1 in BMP signaling in early Xenopus development. EMBO J. 1998; 17:1019-1028.

16. Irie $\mathrm{T}$, Muta $\mathrm{T}$, Takeshige $\mathrm{K}$. TAK 1 mediates an activation signal from toll-like receptor(s) to nuclear factor-kappaB in lipopolysaccharide-stimulated macrophages. FEBS LETT. 2000; 467:160-164.

17. Sato S, Sanjo H, Takeda K, Ninomiya-Tsuji J, Yamamoto M, Kawai T, Matsumoto K, Takeuchi O, Akira S. Essential function for the kinase TAK1 in innate and adaptive immune responses. Nat Immunol. 2005; 6:1087-1095.

18. Hirata Y, Takahashi M, Morishita T, Noguchi T, Matsuzawa A. Post-Translational Modifications of the TAK1-TAB Complex. Int J Mol ScI. 2017; 18.

19. Prabhu L, Mundade R, Korc M, Loehrer PJ, Lu T. Critical role of NF-kappaB in pancreatic cancer. Oncotarget. 2014; 5:10969-10975. https://doi.org/10.18632/oncotarget.2624.

20. Hu E, Ding L, Miao H, Liu F, Liu D, Dou H, Hou Y. MiR30a attenuates immunosuppressive functions of IL-1betaelicited mesenchymal stem cells via targeting TAB3. FEBS LETT. 2015; 589:3899-3907.

21. Roh YS, Song J, Seki E. TAK1 regulates hepatic cell survival and carcinogenesis. J Gastroenterol. 2014; 49:185-194.
22. Criollo A, Niso-Santano M, Malik SA, Michaud M, Morselli E, Marino G, Lachkar S, Arkhipenko AV, Harper F, Pierron G, Rain JC, Ninomiya-Tsuji J, Fuentes JM, et al. Inhibition of autophagy by TAB2 and TAB3. EMBO J. 2011; 30:4908-4920.

23. Jin G, Klika A, Callahan M, Faga B, Danzig J, Jiang Z, Li X, Stark GR, Harrington J, Sherf B. Identification of a human NF-kappaB-activating protein, TAB3. Proc Natl Acad Sci U S A. 2004; 101:2028-2033.

24. Chen J, Gu J, Feng J, Liu Y, Xue Q, Ni T, Wang Z, Jia L, Mao G, Ji L. TAB3 overexpression promotes cell proliferation in non-small cell lung cancer and mediates chemoresistance to CDDP in A549 cells via the NF-kappaB pathway. Tumour Biol. 2016; 37:3851-3861.

25. Ding J, Huang S, Wang Y, Tian Q, Zha R, Shi H, Wang Q, Ge C, Chen T, Zhao Y, Liang L, Li J, He X. Genome-wide screening reveals that miR-195 targets the TNF-alpha/ NF-kappaB pathway by down-regulating IkappaB kinase alpha and TAB3 in hepatocellular carcinoma. Hepatology. 2013; 58:654-666.

26. Zhao N, Wang R, Zhou L, Zhu Y, Gong J, Zhuang SM. MicroRNA-26b suppresses the NF-kappaB signaling and enhances the chemosensitivity of hepatocellular carcinoma cells by targeting TAK1 and TAB3. Mol Cancer. 2014; 13:35.

27. Tao T, He Z, Shao Z, Lu H. TAB3 O-GlcNAcylation promotes metastasis of triple negative breast cancer. Oncotarget. 2016; 7:22807-22818. https://doi.org/10.18632/ oncotarget.8182.

28. Morlon A, Munnich A, Smahi A. TAB2, TRAF6 and TAK1 are involved in NF-kappaB activation induced by the TNFreceptor, Edar and its adaptator Edaradd. Hum Mol Genet. 2005; 14:3751-3757.

29. Ishitani T, Takaesu G, Ninomiya-Tsuji J, Shibuya H, Gaynor RB, Matsumoto K. Role of the TAB2-related protein TAB3 in IL-1 and TNF signaling. EMBO J. 2003; 22:6277-6288.

30. Lee WS, Yun SH, Chun HK, Lee WY, Yun HR, Kim J, Kim K, Shim YM. Pulmonary resection for metastases from colorectal cancer: prognostic factors and survival. Int $\mathrm{J}$ Colorectal Dis. 2007; 22:699-704.

31. Van Cutsem E, Nordlinger B, Adam R, Kohne CH, Pozzo C, Poston G, Ychou M, Rougier P. Towards a pan-European consensus on the treatment of patients with colorectal liver metastases. Eur J Cancer. 2006; 42:2212-2221.

32. Nguyen DX, Bos PD, Massague J. Metastasis: from dissemination to organ-specific colonization. Nat Rev Cancer. 2009; 9:274-284.

33. Valastyan S, Weinberg RA. Tumor metastasis: molecular insights and evolving paradigms. Cell. 2011; 147:275-292.

34. Brunen D, Willems SM, Kellner U, Midgley R, Simon I, Bernards R. TGF-beta: an emerging player in drug resistance. Cell Cycle. 2013; 12:2960-2968. 
35. Romano G, Santi L, Bianco MR, Giuffre MR, Pettinato M, Bugarin C, Garanzini C, Savarese L, Leoni S, Cerrito MG, Leone BE, Gaipa G, Grassilli E, et al. The TGF-beta pathway is activated by 5-fluorouracil treatment in drug resistant colorectal carcinoma cells. Oncotarget. 2016; 7:22077-22091. https://doi.org/10.18632/oncotarget.7895.

36. Goossens-Beumer IJ, Zeestraten EC, Benard A, Christen T, Reimers MS, Keijzer R, Sier CF, Liefers GJ, Morreau H, Putter H, Vahrmeijer AL, van de Velde CJ, Kuppen PJ. Clinical prognostic value of combined analysis of Aldh1, Survivin, and EpCAM expression in colorectal cancer. Br J Cancer. 2014; 110:2935-2944.
37. Yuan R, Wang K, Hu J, Yan C, Li M, Yu X, Liu X, Lei J, Guo W, Wu L, Hong K, Shao J. Ubiquitin-like protein FAT10 promotes the invasion and metastasis of hepatocellular carcinoma by modifying beta-catenin degradation. Cancer Res. 2014; 74:5287-5300.

38. Qiu Y, Yuan R, Zhang S, Chen L, Huang D, Hao H, Shao J. Rock2 stabilizes beta-catenin to promote tumor invasion and metastasis in colorectal cancer. Biochem Biophys Res Commun. 2015; 467:629-637. 